\title{
Sub-millisecond dynamic nuclear spin hyperpolarization in a semiconductor: A case study from $P_{\text {In }}$ antisite in InP
}

\author{
X. J. Wang, Irina Buyanova and Weimin Chen
}

\section{Linköping University Post Print}

N.B.: When citing this work, cite the original article.

Original Publication:

X. J. Wang, Irina Buyanova and Weimin Chen, Sub-millisecond dynamic nuclear spin hyperpolarization in a semiconductor: A case study from $\mathrm{P}_{\text {In }}$ antisite in InP, 2012, Physical Review B Condensed Matter, (86), 20, 205202-1-205202-6.

http://dx.doi.org/10.1103/PhysRevB.86.205202

Copyright: American Physical Society http://www.aps.org/

Postprint available at: Linköping University Electronic Press http://urn.kb.se/resolve?urn=urn:nbn:se:liu:diva-85472 


\title{
Sub-millisecond dynamic nuclear spin hyperpolarization in a semiconductor: A case study from $P_{\text {In }}$ antisite in InP
}

\author{
X. J. Wang, ${ }^{*}$ I. A. Buyanova, and W. M. Chen ${ }^{\dagger}$ \\ Department of Physics, Chemistry and Biology, Linköping University, 58183 Linköping, Sweden \\ (Received 13 June 2012; revised manuscript received 4 November 2012; published 13 November 2012)
}

\begin{abstract}
Optically detected magnetic resonance is employed to identify key factors governing dynamic nuclear polarization (DNP) in a semiconductor. We demonstrate that the extent of DNP can be efficiently controlled by varying lifetime of the localized electrons that transfer spin angular momentum to nuclei. The ultimate speed of a DNP process, on the other hand, is determined by the strength of hyperfine interaction that drives DNP. We show that about $50 \%$ nuclear spin polarization of a $\mathrm{P}_{\mathrm{In}}$ antisite in $\mathrm{InP}$ can be achieved by shortening electron lifetime within a remarkably short time $(<0.1 \mathrm{~ms})$ due to strong hyperfine coupling.
\end{abstract}

DOI: 10.1103/PhysRevB.86.205202

PACS number(s): 76.70.Fz, 71.70.Jp, 72.25.Fe, 76.70.Hb

Due to its long coherence time, nuclear spin is a building block in many proposed novel devices for future spintronics and spin-based quantum computation. ${ }^{1-9}$ In the seminal proposal by Kane, ${ }^{1}$ the nuclear spin of $\mathrm{P}$ shallow donors in $\mathrm{Si}$ was identified as a promising candidate for qubits because the nuclear spin $(I=1 / 2)$ of the $100 \%$ natural abundant isotope ${ }^{31} \mathrm{P}$ offers an isolated two-level system with a well-defined Hilbert space. Quantum information storage time exceeding 100-180 s has been reported in this system. ${ }^{10,11}$ For the NV center in diamond, which is currently the most promising room-temperature spin qubit, nearby ${ }^{13} \mathrm{C}$ nuclear spins can be explored for a controllable quantum register. ${ }^{5}$ The success of these future technologies rests on our ability in efficiently polarizing (initializing), manipulating (computing), and measuring (reading out) nuclear spins. Due to a weak interaction of nuclear spins with external perturbations, the primary means for nuclear spin manipulation is through dynamic nuclear polarization (DNP) via hyperfine (HF) coupling between electron and nuclear spins. This can be achieved by deviating electron spin polarization from its thermal equilibrium by, for example, microwave (MW) saturation or optical pumping, ${ }^{12,13}$ such that subsequent electron spin flips driven by HF interaction towards thermal equilibrium can lead to DNP. Under white light illumination at $1.37 \mathrm{~K}$ and $8.5 \mathrm{~T}$, $68 \%$ nuclear spin polarization $\left(P_{N}\right)$ of $\mathrm{P}$ donors in $\mathrm{Si}$ can be achieved in $\geqslant 150 \mathrm{~s} .{ }^{14}$ By optically pumping hyperfine transitions of a $\mathrm{P}$ donor bound exciton in isotope-enriched ${ }^{28} \mathrm{Si}, 76 \% P_{N}$ has been obtained in $\sim 0.1 \mathrm{~s}$ at $1.4 \mathrm{~K}$ and $425 \mathrm{G} .{ }^{15}$ Unfortunately these time scales are still considerably longer than that desired for quantum computing. Despite their utter importance for spintronics and spin-based quantum computation, critical and outstanding issues on what are the fundamental limits in the degree and ultimate speed of DNP and what measures one can take to extend these limits remain unclear so far. For this, precise knowledge and control of the key physical parameters governing DNP efficiency are required.

Here we demonstrate, by optically detected magnetic resonance (ODMR), ${ }^{16}$ that DNP of a local electron-nuclear $(e-n)$ spin system can be efficiently controlled by manipulating electron lifetime $(\tau)$ via, for example, intercenter charge transfer (ICCT). We further show that the ultimate DNP speed is, on the other hand, determined by HF coupling strength that is inherent to a given $e-n$ spin system. The $\mathrm{P}_{\mathrm{In}}$ antisite in InP is chosen here as a model case because, in its singly positively charged state $\left(\mathrm{P}_{\text {In }}^{+}\right)$, it contains an unpaired electron $\operatorname{spin}(S=1 / 2)$ that interacts with a nuclear $\operatorname{spin}(I=1 / 2)$ of a ${ }^{31} \mathrm{P}$ atom forming the simplest, interacting $e-n$ spin system like $\mathrm{Si}^{: 31} \mathrm{P}$. Therefore knowledge gained from this work could have direct implications on Si:P and other similar spin systems. The electron wave function at $\mathrm{P}_{\mathrm{In}}^{+}$is $s$-like and strongly localized, providing a stronger $\mathrm{HF}$ interaction $(\times 25)$ than $\mathrm{P}$ donors in $\mathrm{Si}$. This could shed light on the effect of HF strength on DNP that has not been explored so far. The electronic and nuclear spin states of $\mathrm{P}_{\mathrm{In}}^{+}$are depicted in Fig. 1(a), where $\uparrow(\downarrow)$ and $\Uparrow(\Downarrow)$ denote electron and nuclear spin directions, respectively. They are described by the spin Hamiltonian:

$$
H=\mu_{B} g \mathbf{B} \cdot \mathbf{S}+A \mathbf{S} \cdot \mathbf{I}-\mu_{n} g_{n} \mathbf{B} \cdot \mathbf{I} .
$$

Here the first and second terms describe electronic Zeeman and HF interaction, respectively. $\mu_{B}$ is the Bohr magneton and $\mathbf{B}$ is an external magnetic field. Both electron $g$ factor and HF parameter of the ${ }^{31} \mathrm{P}$ nuclei are isotropic, with $g=2.003 \pm$ 0.003 and $A=(981 \pm 20) \times 10^{-4} \mathrm{~cm}^{-1} \cdot{ }^{17-20}$ The last term is nuclear Zeeman interaction, where $\mu_{n}$ is the nuclear magneton and $g_{n}=2.26$ is the nuclear $g$ factor. This term is about three orders of magnitudes weaker than the first two terms in applied B and can be neglected here. The electron and nuclear energy levels calculated by Eq. (1) are shown in Fig. 2(a).

As nuclear spin is conserved during an electron spin resonance (ESR) transition, two HF-split ESR lines are expected for $\mathrm{P}_{\mathrm{In}}^{+}$[Fig. 2(a)]. Each ESR transition monitors a specific nuclear spin state, that is, the low-field and high-field ESR lines are measures of $\mathrm{P}_{\mathrm{In}}^{+}$in the nuclear spin-up and spin-down state, respectively. ${ }^{21}$ Here ESR was performed by the ODMR technique to provide sufficient sensitivity required for the studied thin films. ${ }^{16}$ By combining ESR with optical orientation and photoluminescence (PL), ${ }^{22}$ ODMR offers the advantage of both spin and optical sensitivity. $P_{N}$ can thus be obtained by

$$
P_{N}=\frac{I_{\mathrm{ODMR}}(\Uparrow)-I_{\mathrm{ODMR}}(\Downarrow)}{I_{\mathrm{ODMR}}(\Uparrow)+I_{\mathrm{ODMR}}(\Downarrow)} .
$$

Here $I_{\mathrm{ODMR}}(\Uparrow)$ and $I_{\mathrm{ODMR}}(\Downarrow)$ denote the ODMR intensities of the nuclear spin-up and spin-down states, respectively. The particular recombination processes monitored in ODMR 
(a) Electron and nuclear spin states of $P_{\ln }$ antisite in $\ln P$

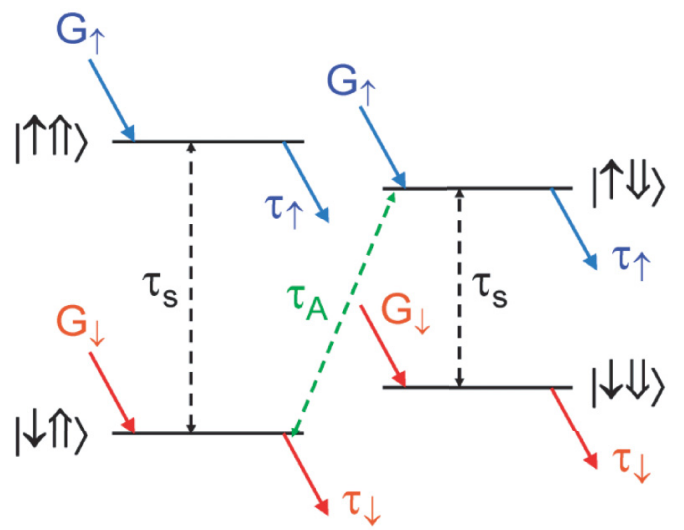

(b) Recombination processes monitored in ODMR

CB

VB

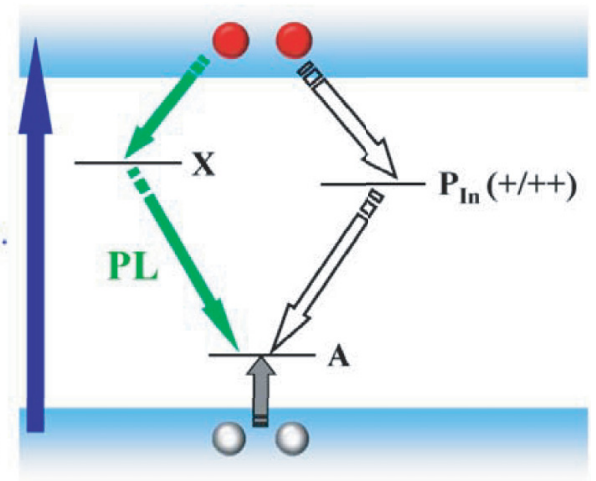

(c) ODMR
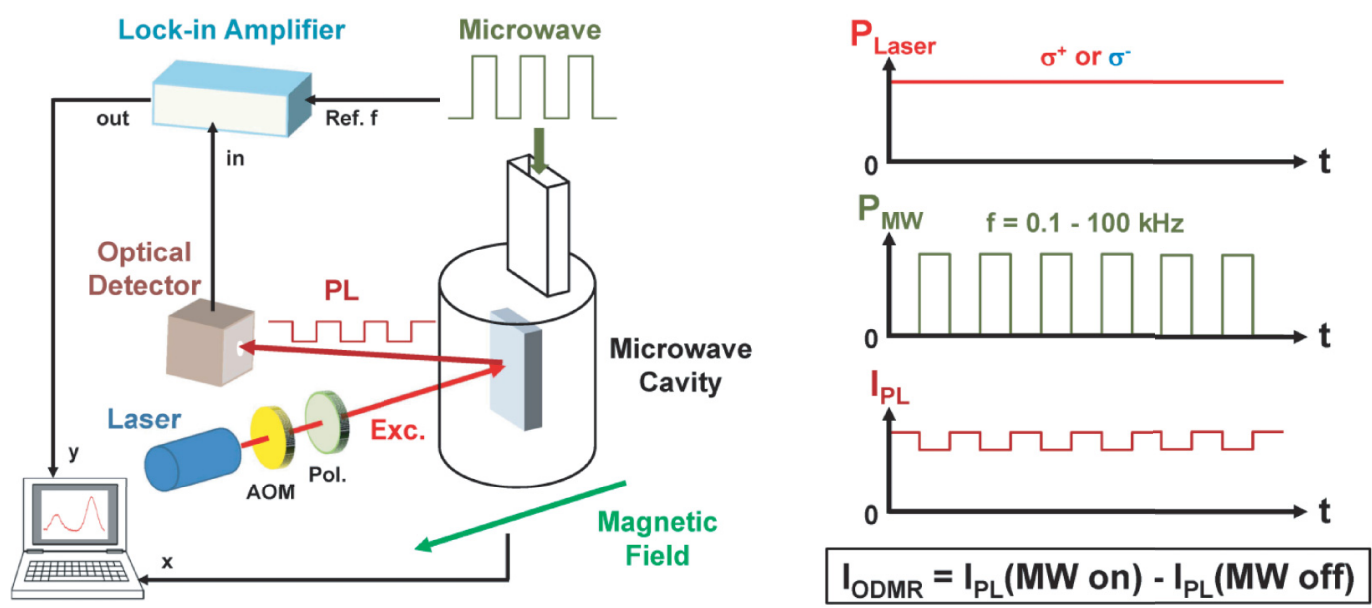

FIG. 1. (Color online) (a) Electron and nuclear spin states of $\mathrm{P}_{\mathrm{In}}^{+}$, together with generation rates $\left(G_{\uparrow}\right.$ and $\left.G_{\downarrow}\right)$ and lifetimes $\left(\tau_{\uparrow}\right.$ and $\left.\tau_{\downarrow}\right)$ of the spin-up and spin-down electron, as well as electron spin relaxation times ( $\tau_{s}$ and $\tau_{A}$ ) that conserves and flips nuclear spin. (b) Competing carrier recombination processes involving $\mathrm{P}_{\mathrm{In}}^{+}$monitored in ODMR. (c) Schematic diagram and modulation scheme of the ODMR experiments.

are schematically shown in Fig. 1(b). $\mathrm{P}_{\text {In }}$ participates in a nonradiative recombination channel of photoexcited electronhole pairs (i.e., ICCT between $\mathrm{P}_{\mathrm{In}}^{+}$and $A$ center) that competes with the radiative recombination process giving rise to a $\mathrm{PL}$ band peaking at $\sim 0.8 \mathrm{eV}$ (i.e., ICCT between the $X$ and $A$ center). ${ }^{19,21}$ If an ESR transition accelerates the former, it will result in a corresponding reduction in the PL intensity ( $\left.I_{\mathrm{PL}}\right)$ that can be detected by ODMR. ${ }^{19,23}$

The studied InP thin films $(\sim 1 \mu \mathrm{m})$ were grown by gas-source molecular beam epitaxy on a (001) InP substrate. The $\mathrm{P}_{\text {In }}$ concentration was $\sim(4-18) \times 10^{16} \mathrm{~cm}^{-3} \cdot{ }^{17,18}$ ODMR was carried out at $12 \mathrm{~K}$ and a MW frequency of $35 \mathrm{GHz}$, see Fig. 1(c). PL was excited by circularly (or linearly) polarized light from a Ti:sapphire laser through optical polarizers, propagating along the direction of $\mathbf{B}$ normal to the sample surface. ODMR signals were detected as ESR-induced $I_{\mathrm{PL}}$ changes by the lock-in technique in phase with on-off MW modulations at 0.1-100 kHz. In time-resolved experiments, an acousto-optic modulator (AOM) was employed to pulse laser beam.

Optical orientation was performed under circularly polarized photoexcitation at $850 \mathrm{~nm}$, which can generate spin polarization of conduction band $(\mathrm{CB})$ electrons up to $50 \% .^{24}$
As generation rate of electron population at $\mathrm{P}_{\mathrm{In}}^{+}$(through capture of an $\mathrm{CB}$ electron by $\mathrm{P}_{\mathrm{In}}^{++}$) is linearly proportional to $\mathrm{CB}$ electron concentration for each spin orientation, electron spin populations at $\mathrm{P}_{\text {In }}^{+}$can be driven off balance leading to a deviation of electron spin polarization $\left(P_{e}\right)$ from its thermal equilibrium value. The steady-state $P_{e}$ is governed by a detailed balance between all relevant processes at $\mathrm{P}_{\text {In }}^{+}$ [Fig. 1(a)] and can be obtained from an analysis of coupled rate equations including generation rates $\left(G_{\uparrow}\right.$ and $\left.G_{\downarrow}\right)$ and lifetimes $\left(\tau_{\uparrow}\right.$ and $\tau_{\downarrow}$ ) of the spin-up and spin-down electron, as well as electron spin relaxation times $\tau_{\mathrm{s}}\left(\tau_{A}\right)$ that conserves (flips) nuclear spin. ${ }^{21}$ Whenever electron spin distribution $n_{\uparrow \Downarrow /} / n_{\downarrow \Uparrow}$ deviates from thermal equilibrium $\beta=\frac{1+e^{-\Delta E / k T}}{1+e^{\Delta E / k T}}$, direct $e-n$ spin flip-flops $|\uparrow \downarrow\rangle \leftrightarrow|\downarrow \uparrow\rangle$, driven by the nonsecular HF interaction $\frac{A}{2}\left(S_{+} I_{-}+S_{-} I_{+}\right)$and described by $\tau_{A}$, will take place leading to DNP of $\mathrm{P}_{\text {In }}^{+}$. (Here $\Delta E$ denotes the electron spin splitting and $n_{i j}$ is the population of $\mathrm{P}_{\mathrm{In}}^{+}$ with the electron and nuclear spin orientations given by $i$ and $j$.)

To manipulate $\tau$ at $\mathrm{P}_{\text {In }}^{+}$we explore the fact that the transition probability $W$ of ICCT is governed by the distance $R$ between the involved centers through $W \propto \exp (-R / a)$, as commonly 

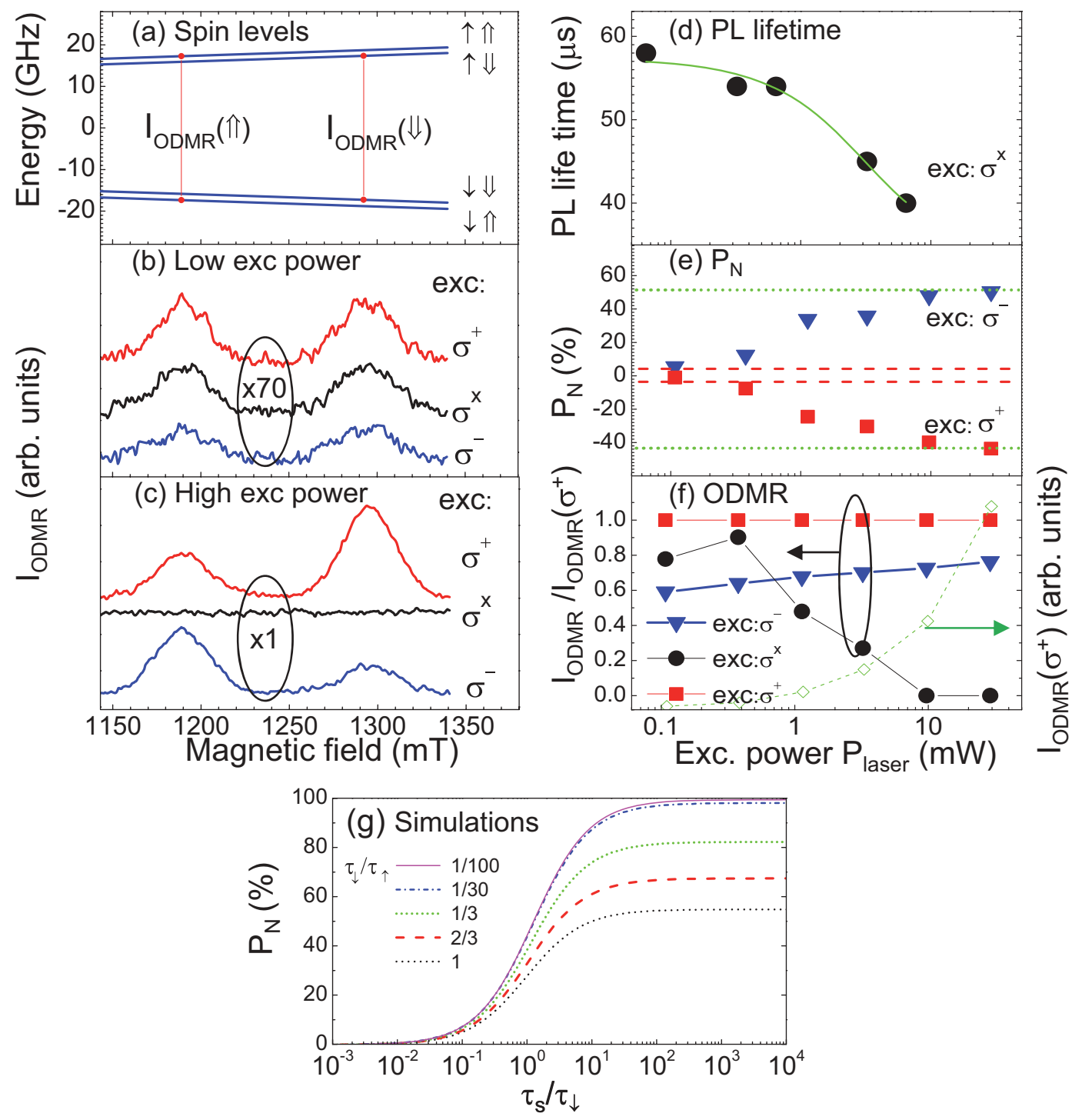

FIG. 2. (Color online) (a) Calculated electron and nuclear spin states from Eq. (1) and the allowed ESR (ODMR) transitions. (b) and (c) ODMR spectra obtained under $\sigma^{+}, \sigma^{-}$, and $\sigma^{x}$ excitation at low and high $P_{\text {Laser }}$. Excitation power dependences of (d) PL decay lifetime, (e) $P_{N}$, and (f) $I_{\mathrm{ODMR}}$ (normalized to that under $\sigma^{+}$excitation) and absolute $I_{\mathrm{ODMR}}$ under $\sigma^{+}$excitation. The experimental error bar for $P_{N}$ is estimated to be about $\pm 2 \%$. The red dashed lines in (e) are calculated $P_{N}$ vs $P_{\text {Laser }}$ near thermal equilibrium, assuming $\tau_{\uparrow}=150 \mu$ s and $\tau_{\uparrow} / \tau_{\downarrow}=0.95$. The simulations for a strongly nonequilibrium case are shown by the green dotted lines in (e), with $\tau_{\uparrow}=1 \mu \mathrm{s}$ and $\tau_{\downarrow}=1.7 \mu \mathrm{s}$ under $\sigma^{+}$excitation that are reversed under $\sigma^{-}$excitation. The other parameters used in the simulations are $\tau_{s}=25 \mu \mathrm{s}, \tau_{A}=10 \mu \mathrm{s}, \tau_{N}=$ $315 \mathrm{~s}$, and $\left|P_{i}\right|=30 \%$. All simulations were obtained from a rate equation analysis, under $\sigma^{-}$(upper curve) and $\sigma^{+}$(lower curve) excitation for each case. (g) Calculated $P_{N}$ as a function of $\tau_{s} / \tau_{\downarrow}$ under $\mathrm{cw} \sigma^{-}$excitation, with several ratios of $\tau_{\downarrow} / \tau_{\uparrow}$, assuming $P_{i}=50 \%, \tau_{s}=25 \mu \mathrm{s}$, $\tau_{A}=10 \mu \mathrm{s}, \tau_{N}=315 \mathrm{~s}$, and $T=12 \mathrm{~K}$.

known for donor-acceptor pair transitions in semiconductors. ${ }^{25}$ Here $a$ denotes the half Bohr radius of the less localized center of the pair. In this work we control $\tau$ by varying excitation laser power $\left(P_{\text {Laser }}\right)$, which effectively changes the occupation of $\mathrm{P}_{\mathrm{In}}^{+}$and $A$, and thus the distance between them. To verify if this is the case, we studied PL decay time [see Fig. 2(d)], which clearly demonstrates the expected control of the ICCT probability by varying $P_{\text {Laser }}$.

Representative ODMR spectra, obtained under circularly $\left(\sigma^{+}\right.$and $\left.\sigma^{-}\right)$and linearly $\left(\sigma^{x}\right)$ polarized excitation at low and high $\mathrm{P}_{\text {Laser }}$, are displayed in Figs. 2(b) and 2(c). The deduced excitation power dependence of $P_{N}$ under $\sigma^{+}$and $\sigma^{-}$excitation is summarized in Fig. 2(e), clearly showing that
$\left|P_{N}\right|$ can be tuned from $\sim 0 \%$ at low $P_{\text {Laser }}$ to $\sim 50 \%$ at the highest $P_{\text {Laser }}$ available.

To provide a quantitative estimate and better understanding of the effect of $\tau$ on $P_{N}$, we carried out a detailed analysis of DNP with the aid of coupled rate equations including all the relevant rates given in Fig. 1(a). ${ }^{21}$ At a very low $P_{\text {Laser }}$ when lifetimes are much longer than electron spin relaxation times, electron spin distribution is mainly governed by the latter and is close to thermal equilibrium with $\frac{n_{\uparrow \Uparrow}}{n_{\downarrow \Uparrow}} \approx \frac{n_{\uparrow \Downarrow}}{n_{\downarrow \Downarrow}} \approx \frac{n_{\uparrow \Downarrow}}{n_{\downarrow \Uparrow}} \approx \beta$. As a result, DNP effect is expected to be very weak as confirmed by $I_{\mathrm{ODMR}}(\Uparrow) \approx I_{\mathrm{ODMR}}(\Downarrow)$ [see Fig. $2(\mathrm{~b})$ ]. The opposite case, that is, when one (or both) of $\tau_{\uparrow}$ and $\tau_{\downarrow}$ is much shorter than $\tau_{s}$ and $\tau_{A}$, applies at a high $P_{\text {Laser }}$ and corresponds to a strongly 
nonequilibrium condition. Now spin population difference is predominantly determined by optical pumping rates and their lifetimes, for example, $\frac{n_{\uparrow j}}{n_{\downarrow j}} \approx \frac{G_{\uparrow} \tau_{\uparrow} n_{0 j}}{G_{\downarrow} \tau_{\downarrow} n_{0 j}}$ for a given nuclear spin state $j$ and $\frac{n_{\uparrow \Downarrow}}{n_{\downarrow \Uparrow}} \approx \frac{G_{\uparrow} \tau_{\uparrow} n_{0 \Downarrow}}{G_{\downarrow} \tau_{\downarrow} n_{0 \Uparrow}}$ across different nuclear spin states. ${ }^{21}$ $\left(n_{0 j}\right.$ denotes the population of $\mathrm{P}_{\mathrm{In}}^{++}$with the given nuclear spin state $j$.) This difference can become very large under $\sigma^{-}$(or $\sigma^{+}$) excitation when $G_{\uparrow} / G_{\downarrow}$ (or $G_{\downarrow} / G_{\uparrow}$ ) can reach as high as 3 , and can be further increased when $\tau_{\uparrow} / \tau_{\downarrow}$ also varies. As $n_{\uparrow \downarrow} / n_{\downarrow \uparrow}$ strongly deviates from thermal equilibrium before DNP takes place (i.e., when $n_{0 \Uparrow}=n_{0 \Downarrow}$ ), the $|\uparrow \Downarrow\rangle \leftrightarrow|\downarrow \uparrow\rangle e-n$ flip-flop process will be active and will drive $n_{\uparrow \Downarrow} / n_{\downarrow \uparrow}$ towards its thermal equilibrium value $\beta$. This forces nuclear spin imbalance towards $\frac{n_{0 \Downarrow}}{n_{0 \Uparrow}} \rightarrow \frac{G_{\downarrow} \tau_{\downarrow}}{G_{\uparrow} \tau_{\uparrow}} \beta$, leading to strong DNP as confirmed experimentally [Figs. 2(c) and 2(e)]. The maximum $P_{N}\left(P_{N}^{\max }\right)$ is limited by $P_{e}$, that is, $\left|P_{N}\right| \leqslant\left|\frac{(1-\beta)+(1+\beta) P_{e}}{(1+\beta)+(1-\beta) P_{e}}\right|$. When $\beta \rightarrow 1$, for example, at a low field and a high temperature desired for practical applications, $P_{N}^{\max } \rightarrow P_{e}$.

Additional support for the direct and important role of $\tau$ on DNP is provided by a close correlation between the $P_{\text {Laser }}$ ranges $(0.3-1 \mathrm{~mW})$ where $\left|P_{N}\right|$ strongly increases [Fig. 2(e)] and $I_{\mathrm{ODMR}}$ under $\sigma^{x}$ excitation $\left[I_{\mathrm{ODMR}}\left(\sigma^{x}\right)\right]$ sharply drops from comparable to $I_{\mathrm{ODMR}}\left(\sigma^{+}\right.$or $\left.\sigma^{-}\right)$to zero [Fig. 2(f)]. The latter can only be explained by a transition of the electron spin distribution at $\mathrm{P}_{\mathrm{In}}^{+}$from near thermal equilibrium to a strongly nonequilibrium condition. At low $P_{\text {Laser }}$, the electron spin

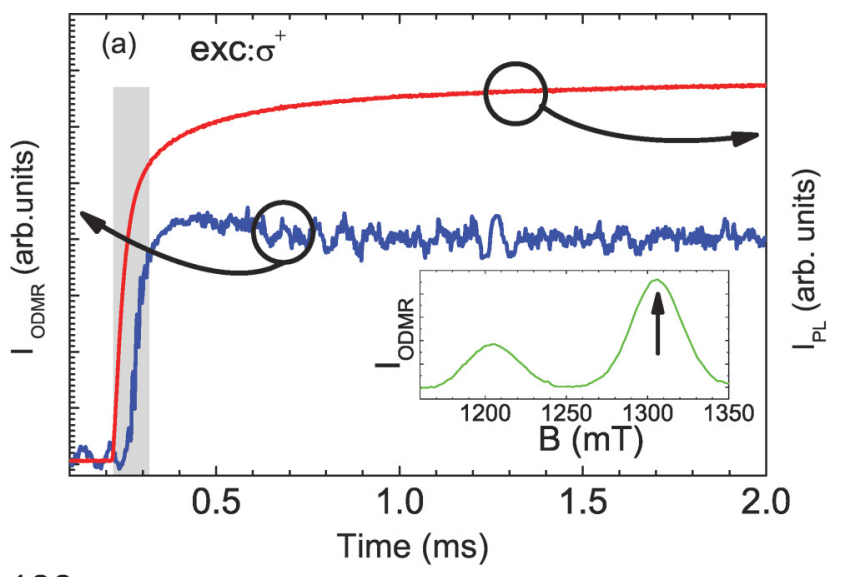

(b)
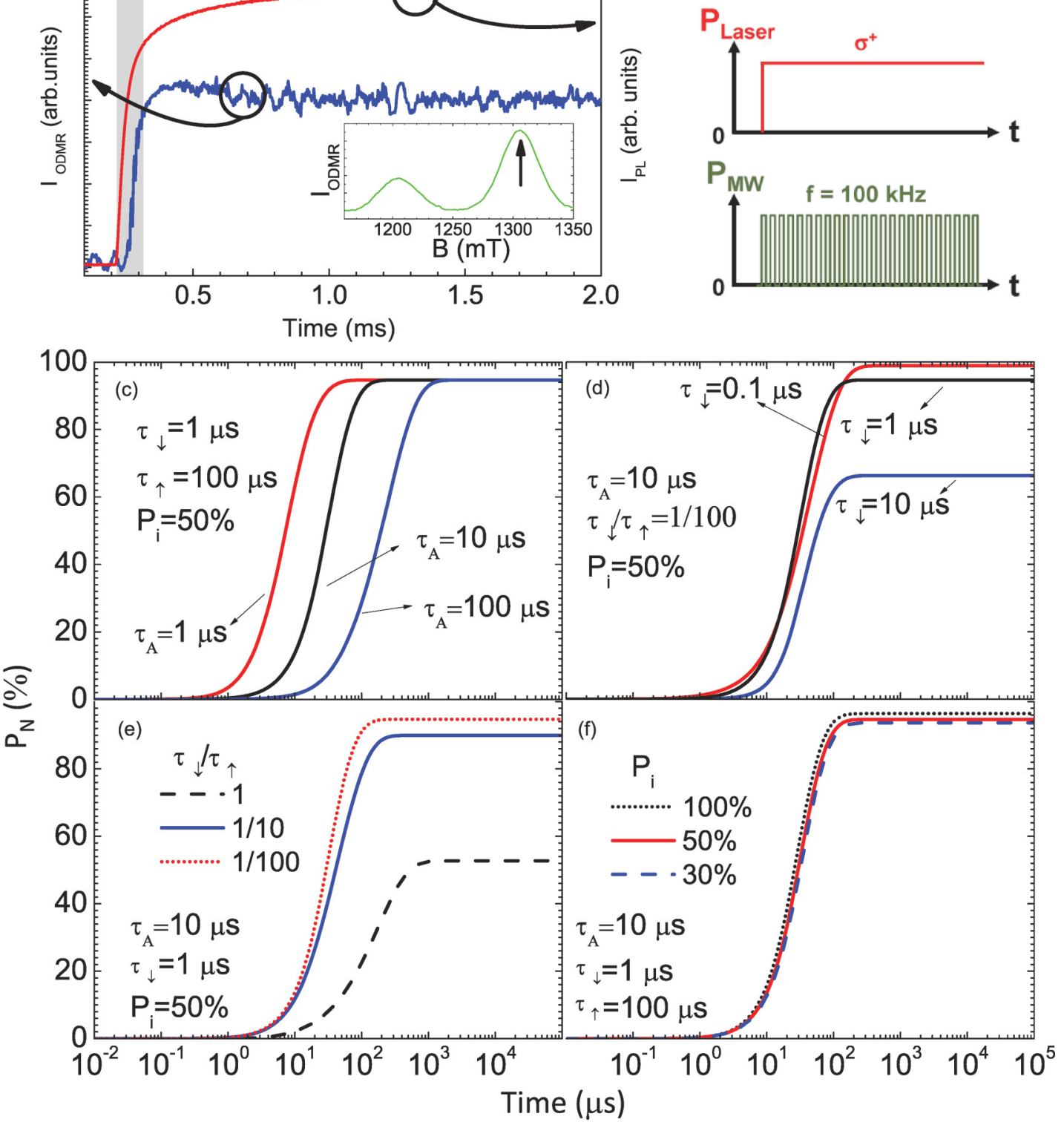

FIG. 3. (Color online) (a) Rising curves of $I_{\mathrm{PL}}$ and $I_{\mathrm{ODMR}}$ immediately after $\sigma^{+}$excitation is switched on by selecting $I_{\mathrm{ODMR}}(\Downarrow)$ as indicated by the arrow in the inset. The instrument response time is represented by the shaded area. (b) The timing of laser and MW pulses. The calculated rising curves of $P_{N}$ after $\sigma^{-}$excitation is switched on are displayed as a function of (c) $\tau_{A}$, (d) $\tau_{\downarrow}$, (e) $\tau_{\downarrow} / \tau_{\uparrow}$, and (f) $P_{i}$, with $\tau_{s}=25 \mu$ s, $\tau_{N}=315 \mathrm{~s}$, and $T=12 \mathrm{~K}$. 
distribution is near thermal equilibrium and is rather insensitive to excitation polarization. As $I_{\mathrm{ODMR}}$ is directly proportional to the spin population difference, ${ }^{21}$ comparable $I_{\text {ODMR }}$ is expected under differently polarized excitation as seen in Fig. 2(b). If $\tau$ is not affected by $P_{\text {Laser }}$, the relative ODMR intensities between differently polarized excitations should remain the same with increasing $P_{\text {Laser }}$. This is in contradiction with our experimental results shown in Fig. 2(f). The observed sharp decrease of $I_{\mathrm{ODMR}}\left(\sigma^{x}\right)$ at high $P_{\text {Laser }}$, despite a strong increase in $I_{\mathrm{ODMR}}\left(\sigma^{+}\right)$and $I_{\mathrm{ODMR}}\left(\sigma^{-}\right)$, can then only be explained if one or both of $\tau_{\uparrow}$ and $\tau_{\downarrow}$ becomes much shorter than $\tau_{s}$ and $\tau_{A}$ leading to a strongly nonequilibrium condition.

In principle, DNP as a dynamic process should increase with increasing $P_{\text {Laser }}$ due to faster turnaround in the optical cycle that induces DNP. To examine the extent of such contribution, we performed a rate equation analysis of DNP. As an example, the calculated dependencies of $P_{N}$ on $P_{\text {Laser }}$ are displayed in Fig. 2(e) by the dashed and dotted lines assuming a constant $\tau$ as determined under the lowest and highest $P_{\text {Laser }}$, respectively. As it can be seen, $P_{N}$ is expected to be independent of $P_{\text {Laser }}$. This is because $P_{N}$ has readily reached its saturation value of the dynamic process permitted for each given $\tau$ even at the lowest $P_{\text {Laser }}$ used $(\sim 0.1 \mathrm{~mW})$, due to fast DNP (see below) combined with efficient electron generation at $\mathrm{P}_{\mathrm{In}}^{+} \cdot{ }^{21}$ The observed sharp increase in $\left|P_{N}\right|$ with increasing $P_{\text {Laser }}$ can thus only be explained by shortening of $\tau$.

To further confirm the effect of $\tau$ on DNP and the fundamental limit in $P_{N}$, we have calculated steady-state $\left|P_{N}\right|$ degree from a rate equation analysis as a function of $\tau$. The trend of increasing $\left|P_{N}\right|$ with decreasing $\tau$ [Fig. 2(g)] conforms to our experimental results. It also confirms that $P_{N}^{\max }$ is limited by $P_{e}$, which critically depends on the ratio of $\tau_{\downarrow} / \tau_{\uparrow}$. It varies, for example, from $P_{N}^{\max }=50 \%$ when $\tau_{\uparrow}=\tau_{\downarrow}$ to $P_{N}^{\max }$ $\rightarrow 100 \%$ when $\tau_{\downarrow} \ll \tau_{\uparrow}$ under $\sigma^{-}$excitation, assuming $P_{i}=$ $\frac{G_{\uparrow}-G_{\downarrow}}{G_{\uparrow}+G_{\downarrow}}=50 \% .100 \%\left|P_{N}\right|$ can in principle be obtained if $\tau$ is tuned to be much shorter than the spin relaxation times and is, moreover, strongly spin dependent (i.e., a large difference between $\tau_{\downarrow}$ and $\tau_{\uparrow}$ ) even when $P_{i} \ll 100 \%$. One strategy to achieve this is to intentionally introduce a high concentration of defects/impurities with strongly spin-polarized holes. ${ }^{26}$

To evaluate the ultimate speed of DNP, we have studied the $I_{\text {ODMR }}$ rising of a given nuclear spin state immediately after the laser light is switched on. A typical rising curve is shown in Fig. 3(a) by detecting $I_{\text {ODMR }}(\Downarrow)$ under $\sigma^{+}$excitation with the timing of laser and MW shown in Fig. 3(b). $I_{\mathrm{ODMR}}(\Downarrow)$ is found to instantly reach its steady-state value, with an identical build-up time as $I_{\mathrm{PL}}$ that is limited by the instrument response time of about $0.1 \mathrm{~ms}$. Therefore, the monitored DNP process must be faster than $0.1 \mathrm{~ms}$. This is remarkable as compared with $\geqslant 150 \mathrm{~s}$ under white light illumination or $0.1 \mathrm{~s}$ under resonant excitation of bound excitons required for $\mathrm{Si}^{31} \mathrm{P} .{ }^{14,15}$

Below we shall examine the dominant factor in determining the ultimate speed of DNP — an unexplored issue so far, with the aid of the rate equation analysis. The predicted rising of $P_{N}$ is shown in Figs. 3(c)-3(f) as a function of $\tau_{A}, \tau_{\uparrow}, \tau_{\downarrow} / \tau_{\uparrow}$, and $P_{i}$. It shows that the onset of the DNP rising is predominantly governed by $\tau_{A}$, which determines the ultimate DNP speed. The observed fast rising of DNP at $\mathrm{P}_{\text {In }}^{+}$must then imply that the $e-n$ spin flip-flop time $\tau_{A}$ should be less than $0.1 \mathrm{~ms}$. According to Fermi's golden rule, $1 / \tau_{A} \propto A^{2}$, meaning that stronger electron localization can accelerate DNP. In this regard, a deeplevel defect such as $\mathrm{P}_{\mathrm{In}}^{+}$in InP is more favorable than a shallowlevel impurity like the $\mathrm{P}$ donor in $\mathrm{Si}$. The stronger $\mathrm{HF}$ coupling is thus believed to be largely responsible for the observed drastic difference in the DNP times between these two $e-n$ spin systems. This finding also suggests that the DNP speed of $\mathrm{Si}^{31} \mathrm{P}$ can potentially be increased by engineering electron wave function overlap with the P nucleus. On the other hand, the saturation value of $\left|P_{N}\right|$ after a long time of excitation is found to be nearly independent of $\tau_{A}$, as shown in Fig. 3(c). Instead, it is determined by the parameters governing $P_{e}$, that is, $\tau_{\uparrow}$ and $\tau_{\downarrow}, \tau_{\downarrow} / \tau_{\uparrow}$ and $P_{i}$ as shown in Figs. 3(d)-3(f). This is consistent with the analysis of $P_{N}^{\max }$ under the steady-state condition shown in Fig. 2(g).

In summary, we have demonstrated that DNP of a local $e-n$ spin system can be efficiently controlled by manipulating $\tau$ via, for example, ICCT, by simply varying optical excitation density - an efficient and yet unexplored approach that can push beyond the present boundary of DNP efficiency. We have also identified what determines the fundamental limit in the ultimate speed of DNP - a currently unknown but utterly important issue in quantum computing as it governs the operation speed of a quantum computer employing nuclear spin qubits. Our work thus sheds light on the current problem of slow DNP in the $\mathrm{Si}^{31} \mathrm{P}$ spin system, and cautions that its manipulation time of nuclear spin qubits could be fundamentally limited due to the weak HF strength inherent to the P shallow donor in $\mathrm{Si}$. As the principle demonstrated here should be general and valid for other $e-n$ spin systems in semiconductors, our results could provide a useful guideline for identifying, designing and optimizing suitable $e-n$ spin systems that are capable of efficiently polarizing and fast manipulating nuclear spins for future spintronics and spin-based quantum computation.

We are grateful to $\mathrm{C}$. W. Tu for providing the samples, and to the Swedish Research Council (Grant No. 621-2011-4254) and National Basic Research Program of China (Contract No. 2011CB925604) for financial support.

\footnotetext{
*Present address: National Laboratory for Infrared Physics, Shanghai Institute of Technical Physics, Chinese Academy of Sciences, 200083 Shanghai, China.

†Corresponding author: wmc@ifm.liu.se

${ }^{1}$ B. E. Kane, Nature (London) 393, 133 (1998).

${ }^{2}$ J. M. Kikkawa and D. D. Awschalom, Science 287, 473 (2000).
}

${ }^{3}$ H. Sanada, S. Matsuzaka, K. Morita, C. Y. Hu, Y. Ohno, and H. Ohno, Phys. Rev. Lett. 94, 097601 (2005).

${ }^{4}$ L. Childress et al., Science 314, 281 (2006).

${ }^{5}$ D. M. V. Gurudev et al., Science 316, 1312 (2007).

${ }^{6}$ J. J. L. Morton et al., Nature (London) 455, 1085 (2008).

${ }^{7}$ D. J. Reilly et al., Science 321, 817 (2008).

${ }^{8}$ S. Foletti et al., Nat. Phys. 5, 903 (2009). 
${ }^{9}$ Ł. Cywiński, W. M. Witzel, and S. Das Sarma, Phys. Rev. Lett. 102, 057601 (2009).

${ }^{10}$ D. R. McCamey et al., Science 330, 1652 (2010).

${ }^{11}$ M. Steger et al., Science 336, 1280 (2012).

${ }^{12}$ A. W. Overhauser, Phys. Rev. 92, 411 (1953).

${ }^{13}$ G. Lampel, Phys. Rev. Lett. 20, 491 (1968).

${ }^{14}$ D. R. McCamey, J. van Tol, G. W. Morley, and C. Boehme, Phys. Rev. Lett. 102, 027601 (2009).

${ }^{15}$ A. Yang, M. Steger, T. Sekiguchi, M. L. W. Thewalt, T. D. Ladd, K. M. Itoh, H. Riemann, N. V. Abrosimov, P. Becker, and H. J. Pohl, Phys. Rev. Lett. 102, 257401 (2009).

${ }^{16}$ For a review on ODMR see, e.g., W. M. Chen, Thin Solid Films 364, 45 (2000).

${ }^{17}$ P. Dreszer, W. M. Chen, K. Seendripu, J. A. Wolk, W. Walukiewicz, B. W. Liang, C. W. Tu, and E. R. Weber, Phys. Rev. B 47, 4111 (1993).
${ }^{18}$ W. M. Chen et al., J. Appl. Phys. 76, 600 (1994).

${ }^{19}$ W. M. Chen et al., J. Electron. Mater. 22, 1491 (1993).

${ }^{20}$ W. M. Chen, I. A. Buyanova, A. V. Buyanov, T. Lundstrom, W. G. Bi, and C. W. Tu, Phys. Rev. Lett. 77, 2734 (1996).

${ }^{21}$ See Supplemental Material at http://link.aps.org/supplemental/ 10.1103/PhysRevB.86.205202 for more information on the ODMR approach, the monitored carrier recombination processes, and the rate equation analysis.

${ }^{22}$ X. J. Wang et al., Nat. Mater. 8, 198 (2009)

${ }^{23}$ W. M. Chen and B. Monemar, Appl. Phys. A 53, 130 (1991).

${ }^{24}$ F. Meier and B. P. Zakharchenya, Optical Orientation (NorthHolland, Amsterdam, 1984).

${ }^{25}$ A. T. Vink, J. Luminescence 9, 159 (1974).

${ }^{26}$ Spin-dependent electron decay is determined by spin polarization of the recombining holes, i.e., $\tau_{\uparrow} / \tau_{\downarrow} \propto h_{\uparrow} / h_{\downarrow}$, where $h_{\uparrow}\left(h_{\downarrow}\right)$ denotes population of spin-up (spin-down) holes. 